Article

\title{
Improvement of the Electro-Optical Process in GaAs for Terahertz Single Pulse Detection by Using a Fiber-Coupling System
}

\author{
Adnan Haj Yahya ${ }^{1}$, Nezah Balal ${ }^{1}$, Avi Klein ${ }^{1}$, Jacob Gerasimov ${ }^{1}$ and Aharon Friedman ${ }^{2, *}$ \\ 1 The Schlesinger Family Center for Compact Accelerators, Ariel University, Ariel 4076414, Israel; \\ adnanhy@ariel.ac.il (A.H.Y.); nezahb@ariel.ac.il (N.B.); avik@ariel.ac.il (A.K.); jacobgr@ariel.ac.il (J.G.) \\ 2 Department of Electrical Engineering, University of Ariel, Ariel 4076414, Israel \\ * Correspondence: aharonfr@ariel.ac.il; Tel.: +972-39-066-887
}

check for updates

Citation: Haj Yahya, A.; Balal, N.; Klein, A.; Gerasimov, J.; Friedman, A. Improvement of the Electro-Optical Process in GaAs for Terahertz Single Pulse Detection by Using a FiberCoupling System. Appl. Sci. 2021, 11, 6859. https://doi.org/10.3390/ app11156859

Academic Editors: Yiming Zhu, Alexander Shkurinov and Chao Li

Received: 27 June 2021

Accepted: 23 July 2021

Published: 26 July 2021

Publisher's Note: MDPI stays neutral with regard to jurisdictional claims in published maps and institutional affiliations.

Copyright: (c) 2021 by the authors. Licensee MDPI, Basel, Switzerland. This article is an open access article distributed under the terms and conditions of the Creative Commons Attribution (CC BY) license (https:// creativecommons.org/licenses/by/ $4.0 /)$.

\begin{abstract}
The electro-optical process is a popular method for terahertz radiation detection. Detectors based on the electro-optical process have large bandwidth, and the signal-to-noise ratio (SNR) is relatively high. Further, this detector can be applied to detect high-power signals without using radiation attenuation. This paper presents a method to improve the electro-optic process to $\mathrm{THz}$ radiation detection based on GaAs crystals by coupling the optical output signal into fiber. Results demonstrated an improvement in the signal-to-noise ratio that means an increase in the dynamic range of the electro-optical detector.
\end{abstract}

Keywords: fiber-coupling; THz pulse; electro-optic detection; GaAs crystal; cross-polarization

\section{Introduction}

Terahertz (THz) technology has proven to be quite elusive; this is the electromagnetic spectrum of $\mathrm{THz}$ radiation situated between infrared light and microwave radiation. Therefore, there is no ionization hazard of $\mathrm{THz}$ radiation and penetration through dielectric materials is reasonably good [1]. The atmospheric scattering of $\mathrm{THz}$ radiation is relatively low [2]. Thus, devices based on this wavelength are becoming increasingly important in a diversity of human activity applications [3,4], e.g., security, imaging, biological, communications, medicine, etc. This led to the development of $\mathrm{THz}$ detection systems such as bolometers, which are essentially heat detectors [5]. Although bolometers have large spectral rangeand less noise-equivalent power, they have a long response time [6]. Electrooptic sampling in nonlinear crystals, e.g., ZnTe, GaAs, Gap, Dast, etc, has been investigated. Electro-optic detection is a second-order nonlinear optical process in which an applied electric field induces a refractive index change in nonlinear crystals at $\mathrm{THz}$ frequencies that is proportional to the applied field [7]. Electro-optic detection requires dealing with phase matching issues. Microfabricated antennas on semiconductors measure the $\mathrm{THz}$ pulse but require specific expertise to make them using cleanroom facilities [8]. There is a high priority to using $\mathrm{THz}$ detectors based on the free-space electro-optic process in nonlinear crystals [9]. Due to the diffraction-limited spatial resolution, femtosecond temporal resolution, $\mathrm{THz}$ frequency bandwidth, $\mathrm{mV} / \mathrm{cm}$ field sensitivity, and signal to noise ratio (SNR) are relatively high $[10,11]$. Typically the ZnTe crystals are used for electro-optic sampling to detect ultrashort $\mathrm{THz}$ pulses using Ti: sapphire fs-lasers at a wavelength around $800 \mathrm{~nm}$ lasers [12]. In contrast to usual Ti: sapphire fs-lasers at a wavelength of $800 \mathrm{~nm}$ the fiber lasers at a wavelength of $1550 \mathrm{~nm}$ systems are compact, low-cost, and in the optical communication range. Recently, various researches have examined and offered methods for improving the electro-optic process detection using ZnTe crystals. The researches which have made a comparison between the ZnTe and GaAs crystals represent the free space detection methods without increasing or improving the output 
signal [13]. This paper presents an improvement of electro-optic detection of $\mathrm{THz}$ single pulse by GaAs crystal by using a fiber coupling system. Gallium Arsenide (GaAs) crystals have been employed in $\mathrm{THz}$ radiation detection [14]. The preference for GaAs crystal is because of its detection frequency range, which reaches $6 \mathrm{THz}$. The coupling into fiber provides an easy way to increase the optic output signal, which can increase the dynamic range of the detector. Experiment results show an improvement and increase of the output signal of the electro-optic detector. Further, the coupling of the optical output signal into fiber allows stretching the $\mathrm{THz}$ pulse, which can be done by using the electro-optic process to detect short $\mathrm{THz}$ pulses [15].

\section{Materials and Methods}

Figure 1 shows a schematic diagram of the electro-optic detection system based on the free space detection method. The THz pulses are emitted by the MMW Accelerator at Ariel University. The frequency of these pluses is $0.11 \mathrm{THz}, 10 \mu$ s width, and $5 \mathrm{~kW}$ power [16]. A CW Fiber laser from keysight Technologies model N7748A is used in this experiment as pump laser. The laser was set at $1535 \mathrm{~nm}$ wavelength, $40 \mathrm{~mW}$ power. A manual fiber polarization controller from Thorlabs (Item FPC031) was used to control the polarization of the laser. An Erbium-Doped Fiber Amplifier (EDFA) was used to amplify the laser at $200 \mathrm{~mW}$. A $100 \mathrm{GHz}$ fiber filter (from E-TEK Dynamics), which is appropriate to the $1535 \mathrm{~nm}$ wavelength, was used to filter the noise around $1535 \mathrm{~nm}$ after amplifying the laser. A focus collimator was used to focus the laser beam into the nonlinear crystal in the free space. Two Off-Axis parabolic gold mirrors (from Thorlabs, item MPD399-M03), were used to focus the THz pulse into the nonlinear crystal. A hole was made in one of the mirrors to allow the laser to pass through it.

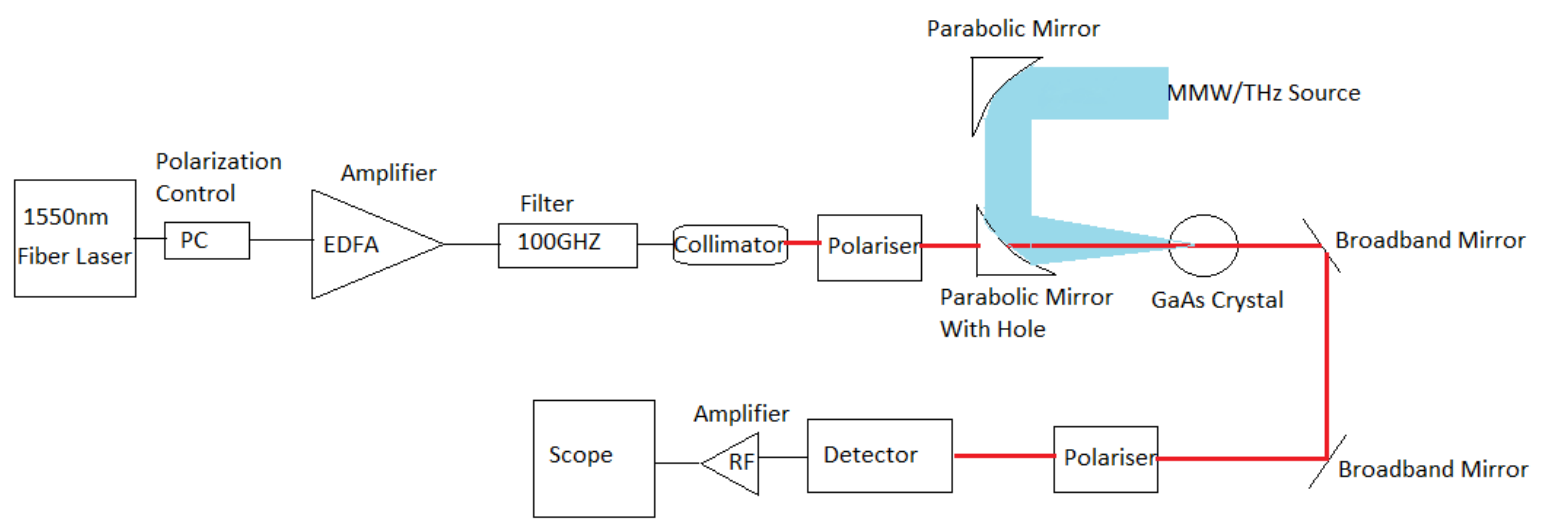

Figure 1. Schismatic diagram for free spice electro-optic detection system.

A GaAs crystal with orientation $<110>$ and $1 \mathrm{~mm}$ thickness was used as a nonlinear crystal. Two Broadband mirrors (from Thorlabs, item BB1-E04) were used to direct the laser beam into the photodetector. Two polarizers applicable to the $1535 \mathrm{~nm}$ wavelength were used to produce a cross-polarization process. The photodetector that used was a Thorlabs, item DET10C. An RF Amplifier (HD communications corp, item HD29225) was used to increase the electric signal of the photodetector at $30 \mathrm{~dB}$. In order to analyze the data an oscilloscope was used (Keysight, item DSOX3104A), with a bandwidth of $1 \mathrm{GHz}$ and a max sample rate of 5 Gigasamples (GSa)/s.

The use of fiber-based systems provides a good method for increasing the optical output signal and improving the signal-to-noise ratio. The use of the EDFA can increase the optical output signal up to $30 \mathrm{~dB}$. The schematic diagram for the electro-optic detection system based on the fiber coupling detection method is shown in Figure 2. The difference between the free space detection system and the fiber coupling detection system is found in the part between the coupling of the laser beam into the fiber and the photodetector. 
A focus collimator was installing in the second polarizer couplingand focused the laser into the fiber. An EDFA was used to amplify the output signal after the coupling system, the output signal was amplified at $30 \mathrm{db}$. Then, the output signal was put through a $200 \mathrm{GHz}$ filter to remove the noise around the increased pulse. A fiber adapter was used to connect the fiber to the photodetector. Then, the RF amplifier increases the electric signal by $30 \mathrm{~dB}$. The data were analyzed by the oscilloscope.

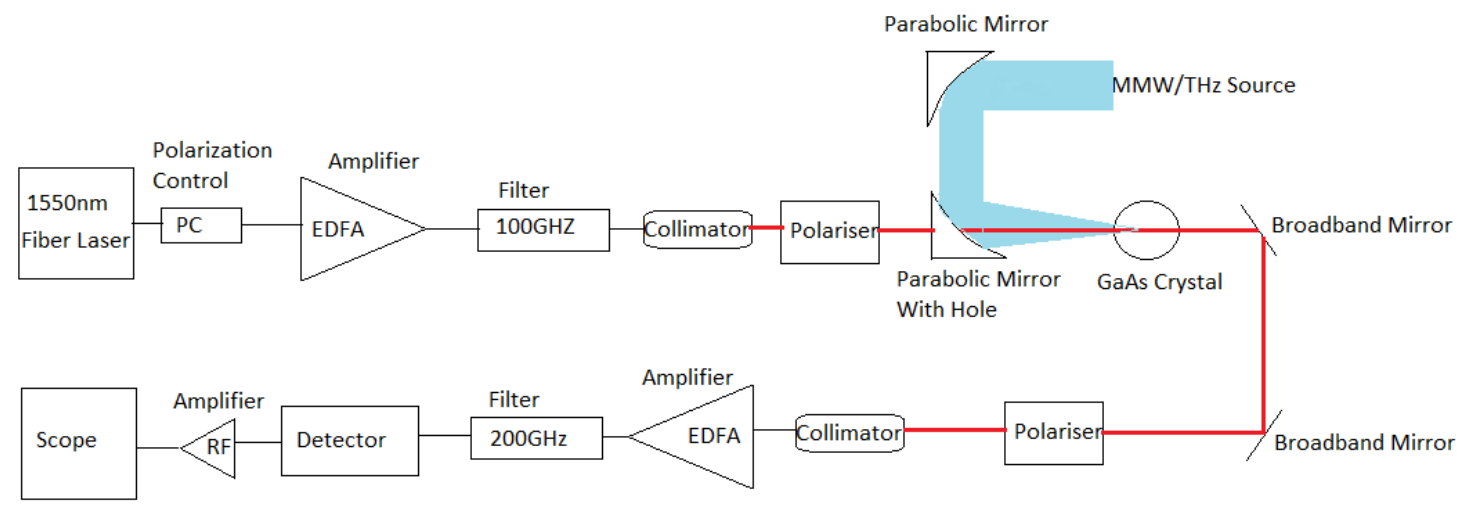

Figure 2. Schismatic diagram for the fiber coupling electro-optic detection system.

\section{Electromagnetic Waves Propagation and Phase-Matching Condition}

The propagation of electromagnetic waves through a crystal is described by the Pockels effect [17]. The polarization of the nonlinear crystal is receipted through the electromagnetic field that is applied to the crystal [18].

$$
P=\chi(E) E
$$

where $P$ is the electric polarization, and is proportional to the applied electric field $E, \chi(E)$ is the electric susceptibility. By expanding $\chi(E)$ in powers of the field $E$ the nonlinear properties of the crystal can be described.

$$
P=\left(\chi_{1}+\chi_{2} E+\chi_{3} E^{2}+\ldots\right) E
$$

where $\chi_{1}$ is the linear susceptibility. The quantities $\chi_{2}$ and $\chi_{3}$ are the second and third-order nonlinear optical susceptibilities, respectively. The second-order nonlinear optical effects are dominant in the nonlinear crystals.

$$
P=E\left(\chi_{1}+\chi_{2} E\right)
$$

The term $\left(\chi_{1}+\chi_{2} E\right)$ is proportional to the electric field. Therefore, a linear dependence is obtained between the electromagnetic field and the refractive index of the crystal. Due to the optimization of nonlinear crystals, the electro-optical (EO) process is a popular method for $\mathrm{THz}$ detection. This process requires a phase-matching condition. This condition is satisfied when the phase of the $\mathrm{THz}$ wave travels at the group velocity of the optical pulse [19]. To obtain the phase matching condition, it is desirable that the coherent length Lc is as long as possible [20].

$$
L c=\frac{c}{2} f\left|n_{T H z}-n_{g}\right|
$$

where $c$ is the speed of light, $n_{T H z}$ is the refractive index in THz pulse, $f$ is THz frequency, and $n_{g}$ is the group index of the optical pulse as defined by [21]

$$
n_{g}=n_{o}-\lambda_{0} \frac{d n_{o}}{d \lambda_{o}}
$$


$\lambda_{o}$ is the optical pulse wavelength and the refractive index $n_{0}$ is defined by

$$
n_{o}^{2}=A+\frac{B \lambda_{o}^{2}}{\lambda_{o}^{2}-c^{2}}
$$

The parameters $A, B, C$ is constants, where $A=8.95 \mu \mathrm{m}, B=2.054 \mu \mathrm{m}$, and $C=0.39 \mu \mathrm{m}[22]$.

\section{Results and Discussion}

In order to perform an effective measurement of the $\mathrm{THz}$ pulse, a phase-matching condition is required, i.e, whether the phase-matching condition in GaAs crystal is obtained in $0.1 \mathrm{THz}$ and $1550 \mathrm{~nm}$ wavelength. The behavior of the $\mathrm{THz}$ refractive index of GaAs and the group refractive index as a function of the frequency $(\mathrm{THz})$ is shown in Figure 3. The blue graph shows the refractive index of GaAs, and the red shows the group refractive index. The $\mathrm{THz}$ refractive index increases exponentially. Figure 3 shows that the refractive index of GaAs is overlapped to the group refractive index up to $6 \mathrm{THz}$, which means the GaAs crystals can obtain phase-matching up to $6 \mathrm{THz}$. Therefore, the phase-matching condition in GaAs crystal is obtained at $0.1 \mathrm{THz}$.

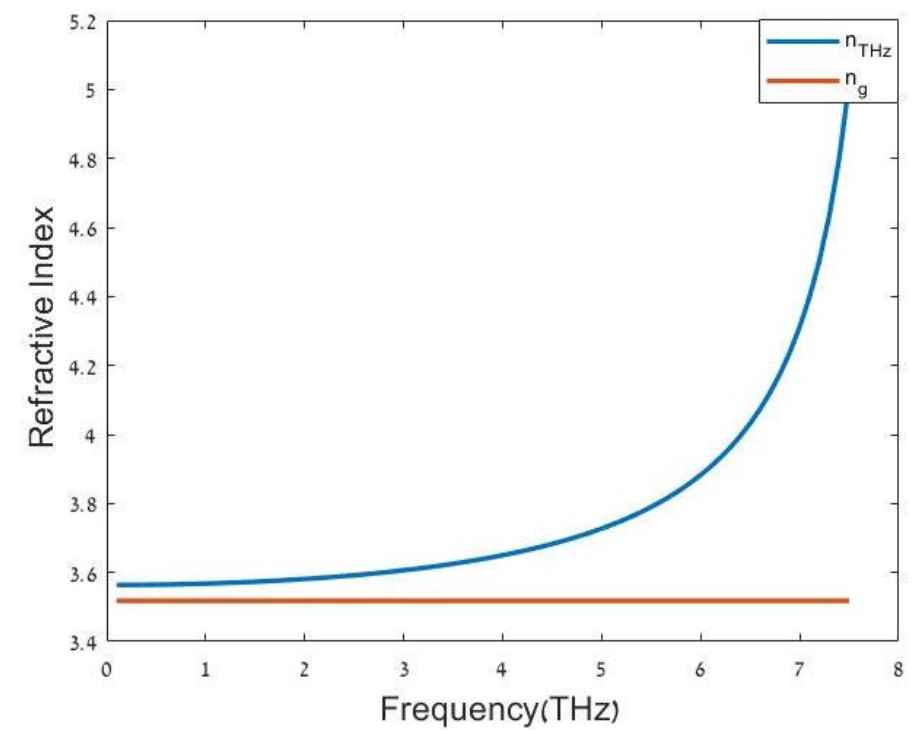

Figure 3. THz refractive index of GaAs and group refractive index.

The wavelength of the used laser should be appropriate to the $\mathrm{THz}$ radiation range to get phase matching in the GaAs crystal for the electro-optic process. The coherent length Lc as a function of laser wavelength concerning $\mathrm{THz}$ radiation is shown in Figure 4. The coherent length should be long as possible to make the electro-optic detection process effective. As the $\mathrm{THz}$ radiation increases, the coherent length decreases as shown in Figure 4. Figure 4 shows that the $1550 \mathrm{~nm}$ wavelength lasers can obtain the phase-matching condition in GaAs crystals for detection of $0.1 \mathrm{THz}$ pulses.

In order to demonstrate an improvement of the electro-optic detection method using GaAs crystals. The THz pulse was detected by two alternative methods: the electro-optic detector system and the Schottky diode detector. Millitech DXP-10 RPFW0 was used as a Schottky diode detector. The maximal safe input to the Schottky diode detector is $40 \mathrm{~mW}$. Therefore the THz pulse was split by a power splitter. The Schottky diode detector receives approximately $30 \mathrm{~dB}$ less than the signal power, and the rest of the pulse power is transmitted to the elector optic detector system. 


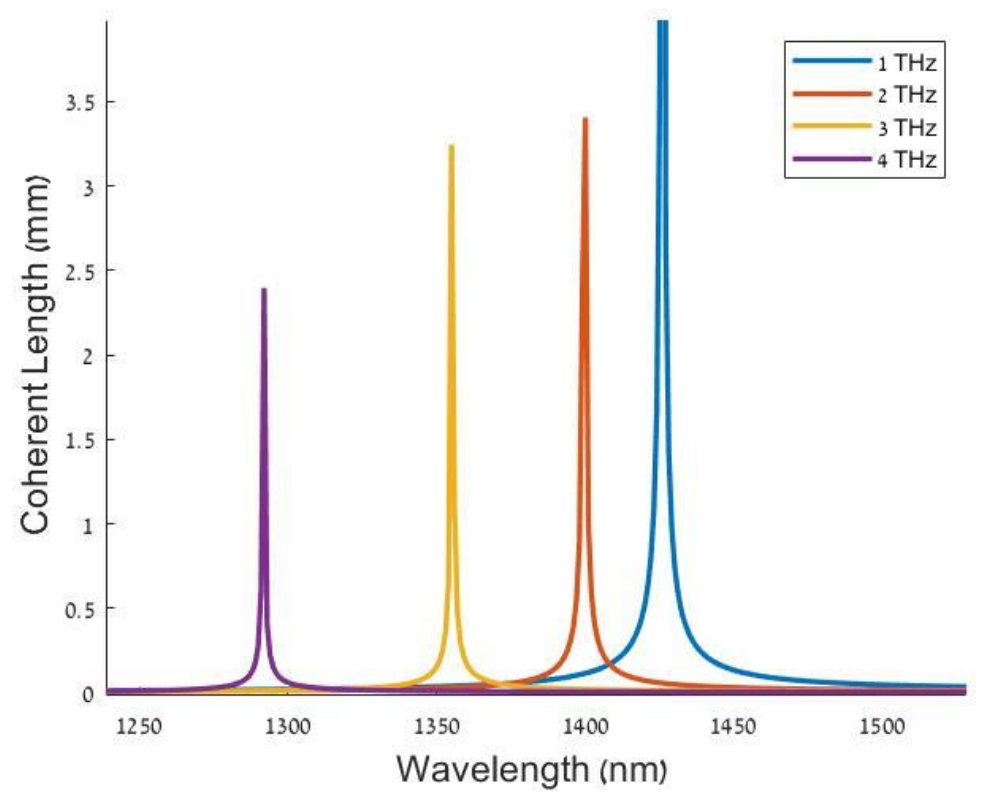

Figure 4. coherence length with respect to $\mathrm{THz}$ frequency.

Figure 5 shows a comparison between the free-space electro-optic detector and the Schottky diode detector. The schematic of the free-space electro-optic system detector is shown in Figure 1; in this experiment, there is no use of fiber coupling. The output signal is detected by a photodetector detector and amplified by an RF amplifier. The blue graph shows the Schottky diode detector. The red graph shows the free space electro-optic detector. Figure 5 shows that the electro-optic detector is close to the noise range and the Schottky diode detector is more clear and quiet. This noise is due to the distortion of the optical output signal in the free space. The output signal can be slightly improved by using a photodetector which has a high gain. The SNR of the free space electro-optic detector in this experiment is $13.9 \mathrm{~dB}$. Although the electro-optic detector is noisy it has a similar response time to the Schottky detector. In order to evaluate the bandwidth improvement in the electro-optic detector, the time duration $\left(\tau_{d}\right)$ of the detection pulse was measured in both of the detectors the Schottky diode and the electro-optic detector. The $\tau_{d}$ of the electro-optic detector is bigger than the Schottky diode by $76.7 \mathrm{ps}$. This indicates that the electro-optic detector has a larger bandwidth than the Schottky diode detector.

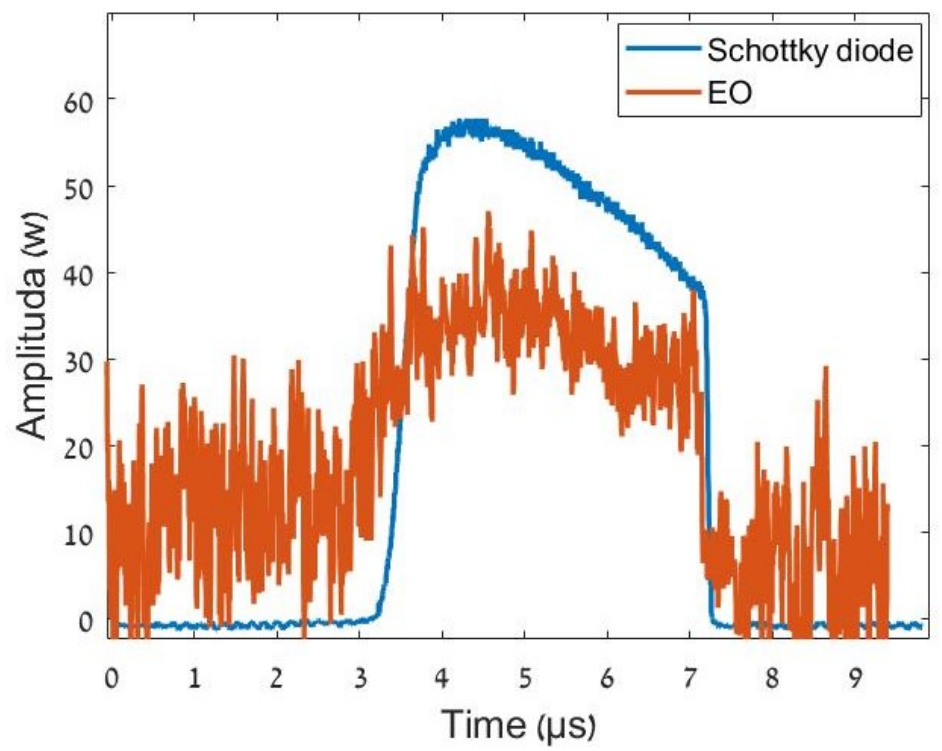

Figure 5. THz pulse detection by free space electro-optic detector system. 
Figure 6 shows a comparison between the fiber-coupling electro-optic detector and the Schottky diode detector. The schematic experiment of the fiber-coupling electro-optic system detector is shown in Figure 2. The blue graph shows the Schottky diode detector. The red graph shows the fiber-coupling electro-optic system detector. Figure 6 shows that the electro-optic detector is more clear and quieter than the Schottky diode detector. Due to the EDFA amplifier and the $200 \mathrm{GHz}$ filter, the laser beam was increased by $30 \mathrm{~dB}$, and the noise around the frequency of the $\mathrm{THz}$ pulse was removed. Which causes significant improvement in the SNR of the output signal of the detector. The SNR of the fiber-coupling electro-optic detector which appears in Figure 6 is $15.66 \mathrm{~dB}$. The $\tau_{d}$ of the electro-optic detector is bigger than the Schottky diode by $135.7 p s$. The $\tau_{d}$ difference between the Schottky diode and the fiber-coupling electro-optic detector almost doubled from the $\tau_{d}$ difference between the Schottky diode and the free-space electro-optic detector. This indicates that the fiber-coupling process was able to increase the bandwidth of the electro-optic detector. Figures 5 and 6 show that the fiber-coupling electro-optic detection system is more clear and quieter than the free space electro-optic detection. This means the fiber-coupling system allows for an increase in the bandwidth and the SNR of the detectors that are based on the electro-optic process. Therefore, the fiber-coupling electro-optic method can present an easy way for detecting short $\mathrm{THz}$ pulses up to $6 \mathrm{THz}$, and 1fs response can be achieved by using a long fiber to stretch the THz pulse [23,24]. The response time of the electro-optic detector can be improved by using a more sensitive and fast photodetector.

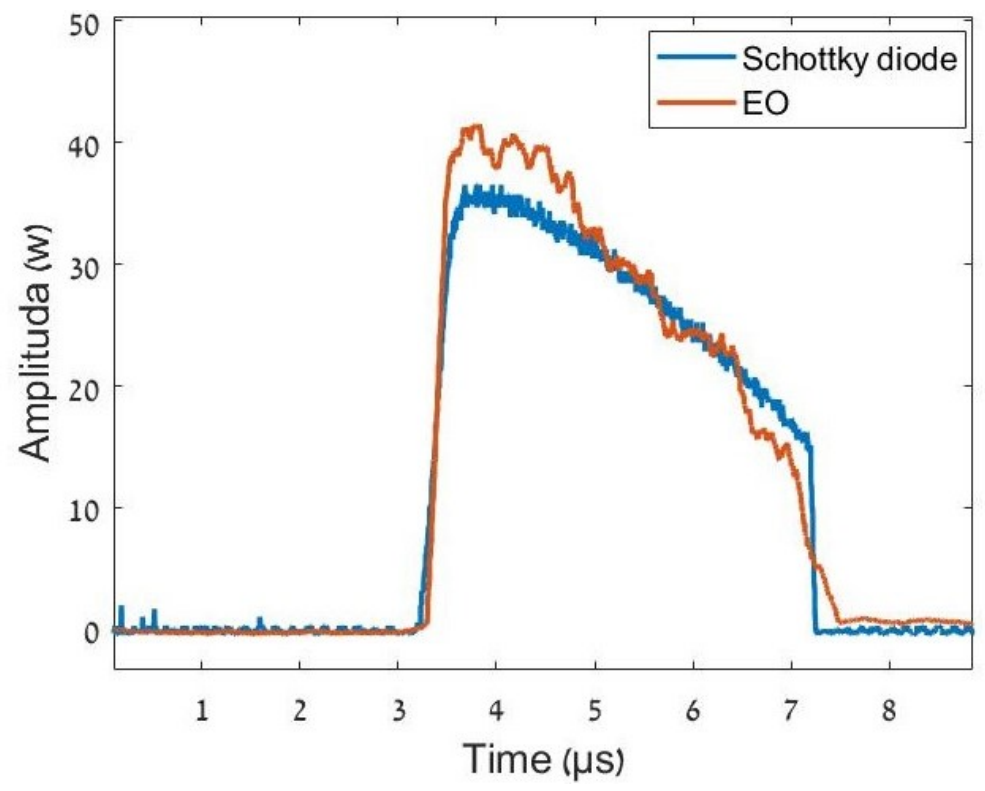

Figure 6. THz pulse detection by fiber coupling electro-optic detector system.

\section{Conclusions}

This paper has presented high-power MMW/THz pulse detection results using the electro-optic effect in GaAs crystal. These results proved that GaAs crystals are an effective detector for $\mathrm{THz}$ pulses. The THz pulse was emitted by the MMW accelerator of Ariel University. The pulse power $0.1-5 \mathrm{~kW}$, radiation frequency of $95-110 \mathrm{GHz}$, and pulse duration of 1-50 $\mu \mathrm{s}$. According to the measuring of the phase matching of the GaAs crystals, they can be used to detect up to $6 \mathrm{THz}$ radiation. Further, the 1550nm wavelength lasers have complied with the terms of the phase matching of the GaAs crystals. This paper presented a new method to improve the use of the electro-optic effect for $\mathrm{THz}$ detection by coupling the output signal into the fiber. The fiber coupling method allows easy access to amplification of the output optical signal, i.e., the laser beam coming out of the GaAs crystal. Therefore, the electro-optic process which includes a fiber coupling system can use to detect short $\mathrm{THz}$ pulses at the ps range. 
Author Contributions: Conceptualization, A.H.Y., Methodology, N.B. and A.H.Y., Data curation, A.K., Investigation J.G., supervision, A.F., Writing-original draft, A.H.Y., All authors have read and agreed to the published version of the manuscript.

Funding: This research was supported by the Schlesinger family center for compact accelerators, Ariel University, Israel.

Institutional Review Board Statement: Not applicable.

Informed Consent Statement: Not applicable.

Data Availability Statement: Data is contained within the article.

Conflicts of Interest: The funders had no role in the design of the study; in the collection, analyses, or interpretation of data; in the writing of the manuscript; or in the decision to publish the results.

\section{References}

1. Haj Yahya, A.; Avi, K. Comparison between Up-Conversion Detection in Glow-Discharge Detectors and the Schottky Diode for MMW/THz High-Power Single Pulse. Appl. Sci. 2021, 11, 4172.

2. Rogalski, A.; Sizov, F. Terahertz detectors and focal plane arrays. Opt. Electron. Rev. 2011, 19, 346-404.

3. Mittleman, D. Sensing with terahertz radiation. In Sensing with Terahertz Radiation; Springer Optical Science 85; Springer: Berlin/Heidelberg, Germany, 2003; pp. 117-153.

4. $\mathrm{Xu}, \mathrm{X}$.; Jingzhou, $\mathrm{X}$. Terahertz wave generation and detection from a CdTe crystal characterized by different excitation wavelengths. Opt. Lett. 2006, 31, 978-980.

5. Hunsche, S.; Kochb, M.; Brenerc, I. THz near-field imaging. Opt. Commun. 1998, 150, $22-26$.

6. Hou, L.; Hi, W. Fast Terahertz Continuous-Wave Detector Based on Weakly Ionized Plasma. IEEE Electron Device Lett. 2012, 52, 1583-1585.

7. Paul, C.; Planken, M. Measurement and calculation of the orientation dependence of terahertz pulse detection in ZnTe. Opt. Soc. Am. 2001, 18, 313-317.

8. Zhao, G.; Schouten, R.N. Design and performance of a THz emission and detection setup based on a semi-insulating GaAs emitter. Rev. Sci. Instrum. 2002, 73, 1715-1719.

9. Zhiping, J.; Xi-Cheng, Z. Terahertz Imaging via Electrooptic Effect. IEEE Transact. Microw. Theor. Tehnic. 1999, 47, 2644-2650.

10. Wu, Q.; Xi-Cheng, Z. Two-dimensional electro-optic imaging of THz beams. Appl. Phys. Lett. 1996, 69, $1026-1028$.

11. Wu, Q.; Xi-Cheng, Z. Ultrafast electro-optic field sensors. Appl. Phys. Lett. 1999, 68, 1604-1606.

12. Pradarutti, B.; Matthäus, G. Highly efficient terahertz electro-optic sampling by material optimization at $1060 \mathrm{~nm}$ Opt. Commun. 2008, 281, 5031-5035.

13. Bang, W.; Lei, C. Comparison of the detection performance of three nonlinear crystals for the electro-optic sampling of a FEL-THz source. Synchrotron Light Sources FELs 2014, 17, 2891-2893.

14. John, F.; Zide, J.M.O.; Gossard, A.C. Enhanced terahertz detection via ErAs:GaAs nanoisland superlattices. Appl. Phys. Lett. 2006, 88, 251119.

15. Evain, C.; Szwaj, C.; Roussel, E. High Repetition-Rate Electro-optic Sampling: Recent Studies Using Photonic Time-Stretch. JACoW Pub. 2017, 121-124.

16. Marks, H.; Gover, A.; Lurie, Y. Enhancement Of Radiative Energy Extraction In An FEL Oscillartor by Post-Saturation Beam Energy Ramping. In Proceedings of the 38th Interntional Free Elctron Laser Conference, Santa Fe, NM, USA, 20-25 August 2017; pp. 244-246.

17. Bartos, C.; Waldow, M.; Matheisen, C. Pockels effect based fully integrated, strained silicon electro-optic modulator. Opt. Express 2011, 19, 17212-17219.

18. Dexheimer, S.L. Principles and Applications. In Terahertz Spectroscopy, 1st ed.; CRC Press Taylor and Francis Group: Boca Raton, FL, USA, 2008; p. 360.

19. Bakker, H.J.; Cho, G.C. Distortion of terahertz pulses in electro-optic sampling. J. Opt. Soc. Am. 2011, 15, 1795-1801.

20. Ajay, N.; Aniruddha, S.W. A wideband coherent terahertz spectroscopy system using optical rectification and electro-optic sampling. Appl. Phys. Lett. 1996, 69, 2321-2323.

21. Casalbuoni, S.; Schlarb, H. Numerical studies on the electro-optic detection of femtosecond electron bunches. Phys. Rev. Spec. Top. 2008, 11, 072802.

22. Masaya, N.; Koichiro, T. Generation and detection of terahertz radiation by electro-optical process in GaAs using $1.56 \mathrm{~mm}$ fiber laser pulses. Appl. Phys. Lett. 2004, 85, 3974-3976.

23. Szwaj, C.; Evain, C.; Le Parquier, M. High sensitivity photonic time-stretch electro-optic sampling of terahertz pulses. Rev. Sci. Instrum. 2016, 87, 103111.

24. Roussel, E.; Evain, C.; Le Parquier, M. A New Scheme for Electro-optic Sampling at Record Repetition Rates: Principle and Application to the First (turn-by-turn) Recordings of THz CSR Bursts at SOLEIL. Proc. IPAC 2014, $2794-2797$. 